\title{
MORTALIDAD PERINATAL. ESTUDIO DE MUESTREO
}

\section{Instituto Małerno Infantil de Bogotá}

\author{
Dr. Arturo Rodríguez Soto

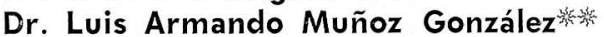

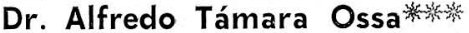

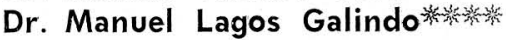

Habiendo analizado las cifras de mortalidad perinatal en los países desarrollados y en los países subdesarrollados, en los cuales las tasas de mortalidad oscilan entre 35 y 50 x $1.000(9,10)$ con posibilidades de sub-registro, y teniendo en cuenta que las cifras altas de mortalidad perinatal son el resultado de condiciones socio-económicas y culturales ( 1 , $2,3,12)$, iniciamos un estudio prospectivo de muestreo en el Instituto Materno Infantil de Bogotá el cual atiende en su mayor parte a pacientes de baja condición socio-económica y que en su mayoría concurren por primera vez a la Institución en el momento del trabajo de parto, cuando ya están en evolución afecciones graves maternas o fetales.

\section{Material y Métodos}

El estudio prospectivo de muestreo se programó en la siguiente forma:

1․ Desde el 1\% de Septiembre de 1974 hasta el 31 de Agosto de 1975, se estudiaron las pacientes que ingresaban cada $5^{\circ}$ día en los turnos diurnos de 12 horas. La muestra fue indiscriminada.
2: Se hizo el seguimiento de los niños nacidos vivos dentro del período perinatal (final del séptimo día) determinando las tasas de mortalidad perinatal básica en sus diversos componentes y según las recomendaciones de la FIGO (6).

3․ Se efectuó además el estudio de mortalidad ante-parto, intraparto, y neonatal temprana determinando las causas básicas de mortalidad según los antecedentes clínicos y el resultado de las autopsias.

4. Se programó practicar autopsias en el $100 \%$ de los niños muertos en el período perinatal.

* Profesor Asociado del Departamento de Ginecología y Obstetricia, Universidad Nacional.

* Profesor Asistente del Departamento de Obstetricia y Ginecoligía, Universidad Nacional.

*** Residente III del Departamento de Ginecología y Obstetricia, Facultad de Medicina, Universidad Nacional.

**** Residente II del Departamento de Ginecolocía y Obstetricia de la Facultad de Medicina, Universidad Nacional.

Este trabajo hace parte del Relato oficial de la Sociedad Colombiana de Obstetricia y Ginecología al XI Congreso Colombiano de Obstetricia y Ginecología. 


\section{Muestra:}

Estuvo constituída por 2.225 productos de los cuales 1.120 (50,34\%) correspondieron al sexo masculino y $1.105(49,66 \%)$ al sexo femenino.

\section{Distribución de las gestantes por edad:}

En el Cuadro N: 1 se muestra que el $81,6 \%$ correspondió a gestantes entre los 15 y 29 años. El grupo menor de 15 años fue de $0,2 \%$ y el grupo de 35 años y más constituyó el $8,2 \%$.

\section{CUADRO N: 1}

NACIMIENTOS $Y$ EDAD MATERNA

$$
\text { I. M. I. }
$$

\begin{tabular}{lrr}
\hline Edad & $\mathbf{N}^{\mathbf{0}}$ & $\%$ \\
\hline$<15$ & 5 & 0.2 \\
$10-19$ & 610 & 27.5 \\
$20-24$ & 821 & 3.9 \\
$25-29$ & 383 & 17.2 \\
$30-34$ & 222 & 10.0 \\
$35-39$ & 133 & 6.0 \\
$40-44$ & 48 & 2.1 \\
$45 y+$ & 3 & 0.1 \\
\cline { 2 - 3 } TOTAL & 2.225 & 100 \\
\hline
\end{tabular}

Distribución de los productos por peso:

En el Cuadro № 2 se hace la distribución por peso de los 2.225 productos que constituyeron la muestra. $14.88 \%$ corresponde a niños con pesos inferiores a 2.500 gramos y el $85.12 \%$ corresponde a niños de más de 2.500 gramos.

\section{Distribución de las pacientes según número de gestación:}

El Cuadro № 3 muestra la distribución de las pacientes de acuerdo al número de gestación. Las primigestantes constituyeron el $41,52 \%$. Las mujeres con 5 gestaciones y más co-
CUADRO Nㄴ 2

NACIMIENTOS $Y$ GRUPOS DE PESOS

I. M. I.

\begin{tabular}{lrr}
\hline Pesos & $N^{\circ}$ & $\%$ \\
\hline & & \\
$1.000-1.499$ & 34 & 1.53 \\
$1.500-1.999$ & 74 & 3.33 \\
$2.000-2.499$ & 223 & 10.02 \\
$2.500-2.999$ & 890 & 40.00 \\
$3.000-3.499$ & 753 & 33.84 \\
$3.500-3.999$ & 217 & 9.75 \\
$4.000-4.499$ & 33 & 1.48 \\
4.500 y + & 1 & 0.05 \\
& 2.225 & 100.00 \\
TOTAL & & \\
& & \\
\hline
\end{tabular}

\section{CUADRO № 3}

NUMERO DE GESTACIONES

\begin{tabular}{rrr|lll} 
G1: & $924:$ & $41.52 \%$ & G7 $:$ & $50:$ & $2.24 \%$ \\
G2: & $483:$ & $21.70 \%$ & G8 $:$ & $45:$ & $2.02 \%$ \\
G3: & $315:$ & $14.15 \%$ & G9 $:$ & $24:$ & $1.07 \%$ \\
G4: & $158:$ & $7.10 \%$ & G10: & $26:$ & $1.16 \%$ \\
G5: & $101:$ & $4.53 \%$ & G1 $1:$ & $14:$ & $0.62 \%$ \\
G6: & $68:$ & $3.05 \%$ & G12: & $17:$ & $0.72 \%$
\end{tabular}

rrespondieron al 14,5\%. Estos datos son importantes para determinar los grupos de alto riesgo.

\section{Mortalidad Perinatal:}

El Cuadro No 4 muestra la tasa de mortalidad perinatal básica en el grupo de 2.225 productos, la cual alcanza a 43,6 x 1.000 y que se descompone en mortinatalidad $16,18 \times 1.000$ y mortalidad neonatal temprana 19.68 $x 1.000$.

La mortinatalidad se distribuyó así: Intraparto $7.64 \times 1.000$ y anteparto $16.18 \times 1.000$. 
Vol. XXVII

No 5

\section{CUADRO N: 4}

COMPONENTES DE LA MORTALIDAD PERINATAL

I.M.I. (muestreo) 1975

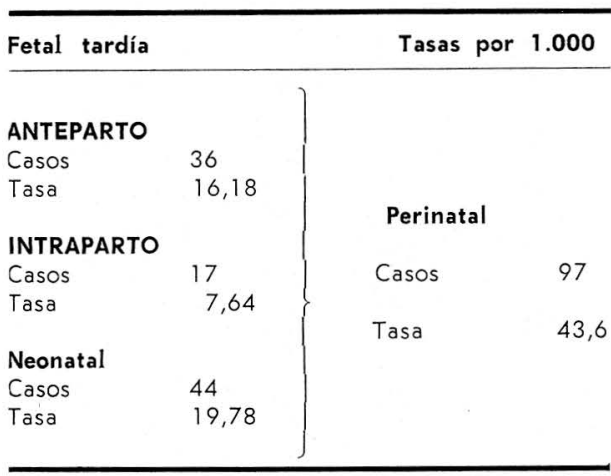

Mortalidad perinatal y peso al nacer:

El Cuadro No 5 muestra la distribución de la mortalidad perinatal por grupos de peso y el desglosamiento de esta tasa en mortalidad fetal tardía y neonatal temprana. Para el grupo de 1.000 a 1.499 gramos la mortalidad perinatal es de $705.88 \times$ 1.000. Para pesos de 1.500 a 1.999 gramos es de 378.37 y 1.000. A partir de este grupo de peso desciende paulatinamente la mortalidad perinatal, encontrándose un nuevo ascenso de $60.60 \times 1.000$ para pesos de 4.000 y más gramos.

En la Gráfica № 1 se muestran los tres componentes de la mortalidad perinatal: intraparto, anteparto $y$ neonatal temprano, siendo evidente que en todos los grupos de peso a excepción del grupo de 2.500 a 2.999 gramos, las muertes anteparto y neonatal tempranas superan ostensiblemente a las muertes intraparto.

\section{Mortalidad perinatal y edad materna}

En el Cuadro № 6 se discriminan las tasas de mortalidad perinatal bá- sica y sus componentes, de acuerdo a la edad materna. Es evidente que el riesgo de muerte perinatal aumenta cuando la edad de la madre es superior a 30 años y se incrementa notoriamente después de los 40 años cuando la tasa de mortalidad perinatal asciende a $62.5 \times 1.000$.

\section{CUADRO N: 6}

MORTALIDAD PERINATAL

$\begin{array}{rc}\text { EDAD MATERNA } & - \text { TASAS POR } 1.000 \\ \text { I.M.I. } & 1975\end{array}$

\begin{tabular}{lccc}
\hline Edades & $\begin{array}{c}\text { Morti- } \\
\text { natalidad }\end{array}$ & $\begin{array}{c}\text { Mortalidad } \\
\text { neonatal }\end{array}$ & $\begin{array}{r}\text { Mortalidad } \\
\text { perinatal }\end{array}$ \\
\hline & & & \\
$15-19$ & 19,67 & 22,95 & 42,62 \\
$20-24$ & 21,92 & 17,05 & 38,97 \\
$25-29$ & 20,11 & 13,05 & 39,16 \\
$30-34$ & 18,01 & 40,54 & 58,55 \\
$35-39$ & 45,12 & 7,51 & 52,63 \\
$40-44$ & 62,50 & 0,00 & 62,50 \\
$45 y+$ & 333,33 & 0,00 & 333,33 \\
\end{tabular}

\section{Mortalidad perinatal y orden de nacimiento}

En el Cuadro № 7 y Gráfica № 2 se aprecian que el riesgo de mortalidad perinatal más bajo corresponde al tercer nacimiento y aumenta en el $4 \circ, 5^{\circ}$ y $6^{\circ}$ y se incrementa notoriamente a partir del $7^{\circ}$ nacimiento cuando las tasas sobrepasan el $80 \times$ 1.000. Esto nos demuestra que la multiparidad se constituye en un factor de riesgo muy elevado.

Es importante mencionar que hasta el cuarto embarazo la mortinatalidad y la mortalidad neonatal temprana guardan equilibrio. A partir del 5: nacimiento la mortinatalidad es notoriamente superior a la mortalidad neonatal temprana. 


\section{Mortalidad Perinatal}

\begin{tabular}{|c|c|c|c|}
\hline peso & $\begin{array}{c}\text { fetal } \\
\text { tardía }\end{array}$ & $\begin{array}{c}\text { neo- } \\
\text { natal } \\
\text { tempr. }\end{array}$ & $\begin{array}{c}\text { peri- } \\
\text { natal }\end{array}$ \\
\hline $1000 \cdot 1499$ & 323,52 & 382,36 & 705,88 \\
\hline $1500 \cdot 1999$ & 162,15 & 21622 & 378,37 \\
\hline $2000 \cdot 2499$ & 22,41 & 26.91 & 49,32 \\
\hline $2500 \cdot 2999$ & 17,97 & 8,99 & 20,96 \\
\hline $3000 \cdot 3499$ & 9,29 & 0, & 9,29 \\
\hline $3500 \cdot 3999$ & 0, & 4.60 & 4,60 \\
\hline $4000 \cdot 4499$ & 60,60 & 0, & 60,60 \\
\hline
\end{tabular}

CUADRO $\mathrm{N}^{\circ} 5$

MORTALIDAD PERINATAL $Y$ PESO AL NACER

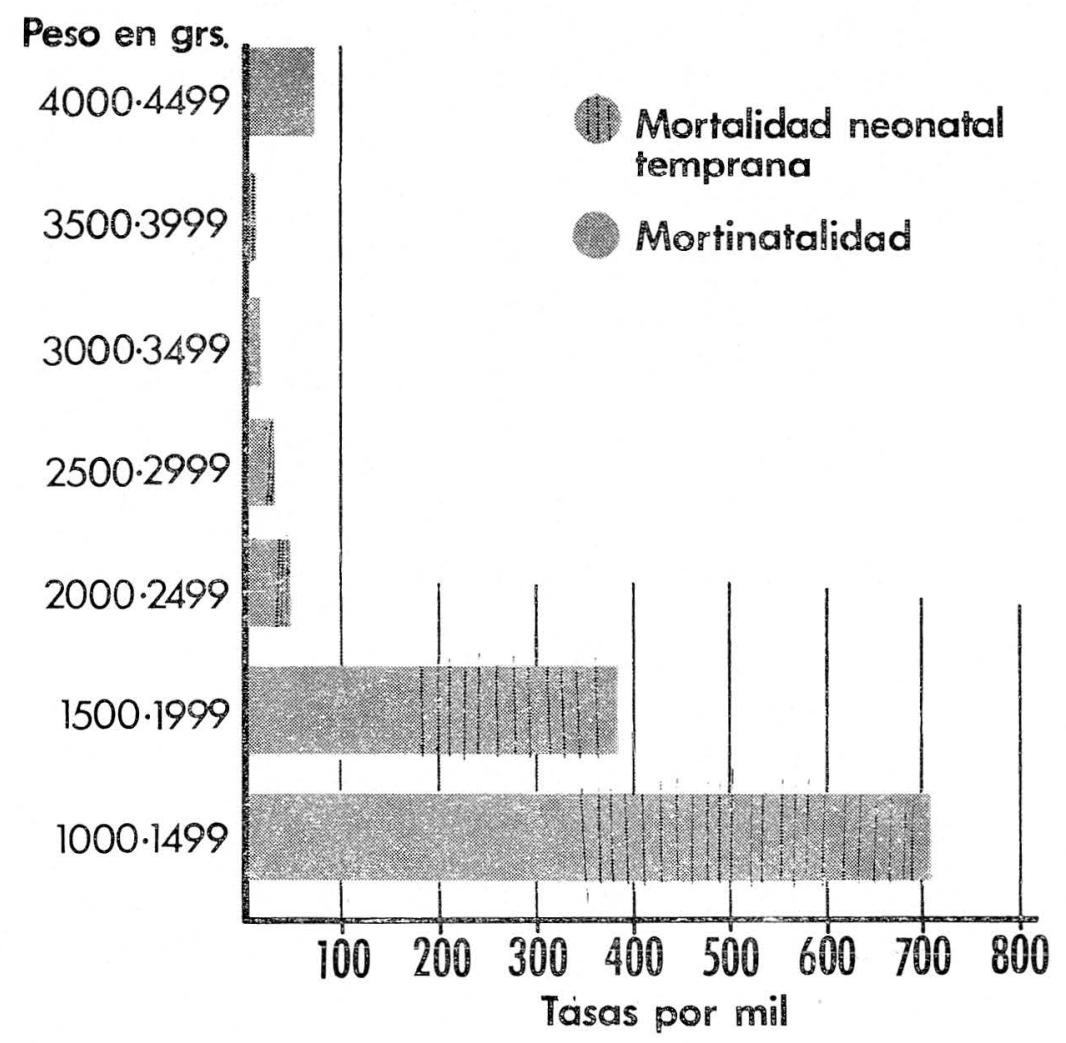

GRAFICA N $N^{0} 1$ 


\section{Mortalidad perinatal y orden de nacimiento}

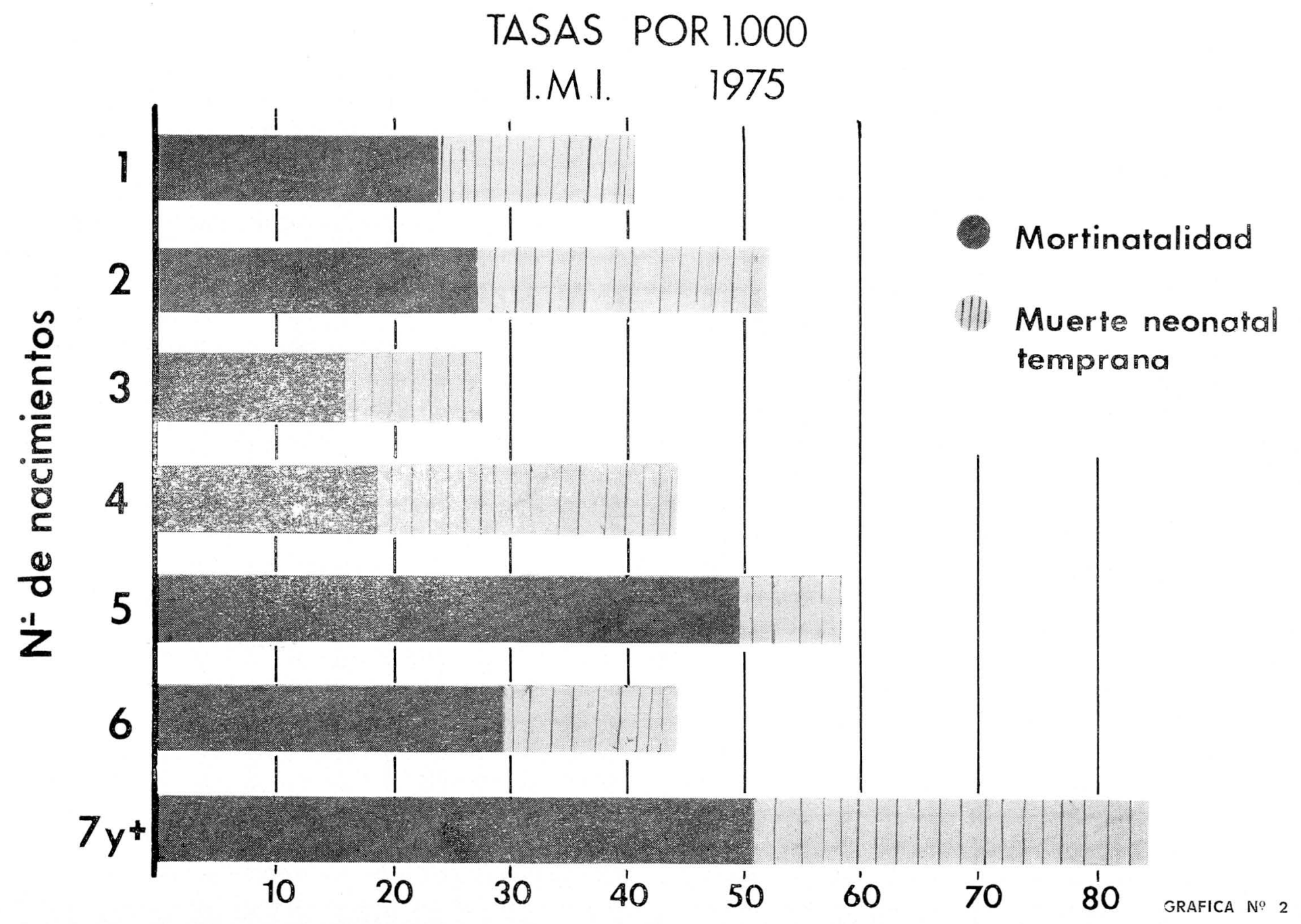


CUADRO № 7

MORTALIDAD PERINATAL

$Y$ ORDEN DE NACIMIENTO

TASAS POR 1.000

I.M.I.

1975

\begin{tabular}{lccc}
\hline Orden & $\begin{array}{c}\text { Morti- } \\
\text { natalidad }\end{array}$ & Neonatal & Perinatal \\
\hline & & & \\
1 & 23,81 & 17,31 & 41,12 \\
2 & 20,70 & 24,84 & 45,54 \\
3 & 15,88 & 12,69 & 28,57 \\
4 & 18,99 & 25,31 & 44,30 \\
5 & 49,50 & 9,9 & 59,40 \\
6 & 29,41 & 14,70 & 44,11 \\
$7 y+$ & 50,08 & 30,48 & 80,56 \\
& & & \\
\hline
\end{tabular}

\section{Causas de muerte}

Suelen ser multifactoriales y guardan relación con afecciones maternas, placentarias o fetales.

En este estudio se logró efectuar autopsias en el $80.41 \%$ de los casos cifras que consideramos muy importantes, dada la dificultad de practicar autopsias en niños o fetos muertos en nuestra Institución.

El Cuadro № 8 describe las causas de mortinatalidad discriminadas en el período anteparto e intraparto y las causas de muerte neonatal temprana.

CUADRO № 8

CAUSAS DE LA MORTALIDAD PERINATAL

\begin{tabular}{|c|c|c|c|}
\hline Anteparto & $\%$ & & \\
\hline * Toxemia & 50.0 & & \\
\hline * Anomalías congénitas & 16.7 & & \\
\hline * Hemarragia & 8.3 & Neonatal temprana & $\%$ \\
\hline * Afecciones cordón & 5.5 & & \\
\hline * Sin determinar & 19.5 & * Membrana hialina & 25.0 \\
\hline & & * Neumopatías & 22.7 \\
\hline Intraparto & $\%$ & * Prematurez & 18.4 \\
\hline & & * Hemorragias fetales & 9.0 \\
\hline * Afecciones cordón & 23.5 & * Afecciones cordón y placenta & 6.8 \\
\hline * Anomalías congénitas & 17.7 & * Anomalías congénitas & 15.9 \\
\hline * Hemorragia & 17.7 & * Septicemia & 12.2 \\
\hline * Toxemia & 17.7 & & \\
\hline * Distocias & 11.7 & & \\
\hline * Sin determinar & 11.7 & & \\
\hline
\end{tabular}

\section{Mortalidad anteparto}

Estas defunciones guardan relación directa con la toxemia gravídica, las anomalías congénitas incompatibles con la vida y las hemorragias anteparto.

La tasa de mortalidad anteparto fue de $18.8 \times 1.000$; en el $50 \%$ de estas muertes solo se encontró como antecedente la toxemia gravídica; en el $16,7 \%$ se encontraron anomalías congénitas incompatibles con la vida. Las hemorragias anteparto y la patología del cordón se presentaron en el $13,85 \%$.

En el $19,45 \%$ de los casos no fue posible determinar la causa de la muerte por ser fetos macerados sin ninguna patología aparente, 0 porque no existía en la historia materna ningún antecedente que se pudiera relacionar con la muerte anteparto. 


\section{Mortalidad intraparto}

Tasa $7.64 \times 1.000$. En este grupo se encuentra como causa principal de muerte la anoxia, ocasionada por patología placentaria y del cordón del tipo de la placenta previa, el abruptio, la procidencia del cordón, los nudos, circulares etc. Constituye el $41,18 \%$ de las muertes intraparto y en su gran mayoría se piensa que este grupo de causas pueden ser prevenibles. Siguen en su orden como causas de mortalidad intraparto las anomalías congénitas y la toxemia gravídicá con un $17,7 \%$ para cada una y finalmente los partos distócicos con un $11,7 \%$. No se pudo determinar la causa de la muerte en un $11,7 \%$.

\section{Mortalidad necnatal temprana}

Tasa $19,78 \times 1.000$.

La evolución del recién nacido durante los primeros días de vida está predeterminada en gran parte por factores hereditarios, por el medio materno y por los efectos del parto.

En la evaluación inicial debe tenerse encuenta la adaptación al medio, el peso al nacer, el tiempo de gestación, el estado de nutrición, y la existencia de anomalías congénitas $(9,1$, $2,3,12)$.

El riesgo de muerte aumenta a medida que disminuye el peso al nacer. En el presente estudio ya se comentó que para pesos de 1.000 a 1.499 gramos la mortalidad neonatal temprana es de 382,36 x 1.000; para pesos de 1.500 a 1.999 es de 216,22 x 1.000 y que a partir de este peso hay disminución notoria en las tasas de mortalidad neonatal temprana.

La mayoría de los problemas neonatales se plantean en las 24 horas que siguen al nacimiento; en este pri- mer día suelen surgir problemas importantes como consecuencia de la anoxia intraparto, malformaciones congénitas o complicaciones por insuficiencia ponderal.

Los problemas que suelen plantearse en la primera semana están relacionados con las infecciones, la ictericia neonatal, trastornos del metabolismo, insuficiencia de peso, secuelas de traumatismos obstétricos (2, $4,7,8,11,12)$.

En los países desarrollados se ha logrado reducir la tasa de mortalidad neonatal temprana a cifras menores a $10 \times 1.000$, situación diferente a la de los países en desarrollo que muestran tasas que doblan la anterior (5, 10).

En el presente estudio de muestreo se encontraron como causas principales de muerte neonatal temprana las nєumopatías que incluyen la membrana hialina con $47,7 \%$. Le siguen en su orden la insuficiencia ponderal y las anomalías congénitas con $15,8 \%$. La infección fue causa de muerte neonatal en un $12,2 \%$.

En el $17,15 \%$ de todos los niños a los cuales se les practicó autopsia se encontró algún tipo de anomalía congénita. Estas se distribuyeron así: $52,2 \%$ malformaciones cardíacas, $21 \%$ malformaciones del aparato urinario; $15,7 \%$ malformaciones múltiples y $10,5 \%$ malformaciones del sistema nervioso.

\section{Resumen y Conclusiones}

Se presentan los resultados de un estudio de muestreo de mortalidad perinatal básica efectuado en el Instituto Materno Infantil de Bogotá durante un año.

El índice de mortalidad perinatal básica fue de $43,60 \times 1.000$ que se descompone en mortinatalidad 23,82 
x 1.000 y mortalidad neonatal temprana $19,78 \times 1.000$.

La mortinatalidad se distribuyó en intraparto $7,64 \times 1.000$ y anteparto $16,18 \times 1.000$.

Las cifras estudiadas muestran que hay una definida relación entre peso al nacer, edad de la madre, y orden de nacimiento con la mortalidad perinatal.

Se practicaron autopsias en el $80,41 \%$ de los productos muertos y se encontró que las principales causas de muerte en el período anteparto guardan relación con la toxemia gravídica, las anomalías congénitas y las hemorragias.

Las causas de muerte intraparto están relacionadas principalmente con afecciones de la placenta y el cordón.

Las muertes neonatales tempranas guardan especial relación con neumopatías, insuficiencia ponderal, infecciones y anomalías congénitas.

\section{Summary and Conclusions}

The results of a basic perinatal mortality sampling study done at the Instituto Materno Infantil of Bogotá during a year are put forward.

The basic perinatal mortality index was $43.60 \times 1.000$, divided into 23.82 $\times 1.000$ stillbirths and $19.78 \times 1.000$ neonatal mortality.

Stillbirths were distributed between intrapartum $(7.64 \times 1.000)$ and antepartum $(16.18 \times 1.000)$.

The studied figures show that there is a definite relationship between weight at birth, age of the mother and birth order and perinatal mortality.

Autopsies were practiced to 80.41 per cent of the stillborn and it was found that the main causes for death in the antepartum period are related with toxemia of pregnancy, congenital anomalies and hemorrhages.

The causes for intrapartum death are mainly related to placenta and umbilical cord diseases.

The early neonatal deaths are closely related to pneumopathy, ponderal insufficiency, infections and congenital anomalies.

\section{BIBLIOGRAFIA}

1 ABRAMORIREZ, M. and KASS, E. H.: Terathogenesis and prognosis of prematurity. New Engl. J. Med. 275: 878, 1966.

2 BAIRD, P.: Epidemiology of prematurity. J. Pediat. 65: 909, 1964.

3 BISHOP, E.: Prematurity, etiology and management. Post grad. Med. 35: 185, 1964.

4 DE WITT-GREENE, A. G.: Mortalidad y morbilidad perinatales. Ed. Med. Hosp. Infant. (Mex.) 5: 24, 1968.

5 DIAZ DEL CASTILLO, E.: Perspectivas futuras de la perinatología. Bol. Med. Hosp. Infant. Mex. 30: 97.1973.

6 F.I.G.O. News definition of terms in human reproduction. International Journal of Gynecology \& Obstetrics. 12: 30, 1974.

7 JURADO GARCIA, E.: Epidemiología de la prematurez. Bol. Med. Hosp. Infant. Mex. 27: $277,1970$.

8 MILLER, H. and CONKLIN, E.: Clinical evaluation of respiratory insufficiency in new born infant. Pediatrics. 16: 427, 1965.

9 Organización Mundial de la Salud. La prevención de la morbilidad y de la moltalidad perinatales. Informe técnico No 42. Ginebra, 1972.

10 Organización Mundial de la Salud. Prevención de la mortalidad y morbilidad perinatales. Informe técnico No 457. Ginebra, 1970.

11 PAGE, E.: Patogenia y profilaxis del bajo peso al nacer. Clin. Obstet. y Ginec. Marzo $79,1970$.

12 ROSS, R. A.: Some important factors in perinatal mortality statistics in a rural State. Amer. J. Obstet. Gynec. 88: 342, 1964. 